\title{
Acute non-specific back pain management in the emergency setting: A review of the literature
}

\author{
David W. Gullick, RN, Post Grad Dip Rural Crit Care*
}

Emergency Department, Goulburn Valley Health, Shepparton, Australia

Received 26 July 2007; accepted 15 November 2007

\section{KEYWORDS}

Back pain;

Review;

Emergency

department;

Sciatica

\begin{abstract}
Summary Back pain affects a large number of Australians each year. Patients frequently attend emergency departments with acute episodes of back pain. There is a growing body of evidence regarding management of non-specific back pain. A comprehensive review of the literature was undertaken to identify the current evidence base for management of non specific lower back pain. Clinicians managing patients presenting with lower back pain should exclude high risk illnesses with a diagnostic triage including a thorough history and physical examination. If signs and symptoms of serious pathology are excluded imaging and pathology tests are unwarranted. The literature suggests conservative management with simple analgesia and advice to maintain activity and avoid bed rest results in improved outcomes although a proportion of patients will progress to chronic back pain. Psychosocial and work factors are important in the progression to chronicity and clinicians should be aware of these risk factors. Further research is required to determine the clinical relevance of many aspects of the current management of lower back pain. This review provides an update for clinicians and suggests a conservative approach is appropriate in the management of most acute episodes of back pain presenting to the emergency department.
\end{abstract}

(c) 2007 College of Emergency Nursing Australasia Ltd. Published by Elsevier Ltd. All rights reserved.

\section{Introduction}

Back pain is a relatively common presentation to Australian emergency departments with over $70 \%$ of people in developed countries experiencing low back pain at some stage in their lives. ${ }^{1}$ Low back pain has the potential to severely restrict a patient's functionality and ability to earn a living

\footnotetext{
* Correspondence address: 20 Lagana Drive, Shepparton, Victoria, 3630, Australia. Tel.: +61 358314760 .

E-mail address: pgullick@bigpond.net.au.
}

and causes a great degree of discomfort and pain. Back pain can be difficult to treat in the emergency setting as patients often have high expectations of pain relief and investigation. ${ }^{2}$ The aims of management of lower back pain in the emergency setting are to exclude serious conditions, provide adequate pain relief, appropriate advice and follow up and where necessary conduct further investigations. This review will present current evidence and recommendations for practice on patient assessment, imaging, drug therapy, patient advice and non pharmacological therapies and conclude with implications for practice. The objectives of this study are to review the current evidence base for assessment and treatment of acute low back pain in the 
emergency or primary care setting and to provide guidance to clinicians managing acute non-specific low back pain in the emergency and primary care setting.

\section{Methods}

The review will be limited to patients with acute (less than three months) non specific low back pain. Non specific low back pain is defined as pain in the lower back not attributable to a recognisable pathology with or without associated leg pain (sciatica). ${ }^{3}$ Therapies and interventions for conditions such as neoplasms, fracture, infection, spinal cord stenosis, cauda equina, ankylosing spondylitis and degenerative disc diseases will not be discussed. Specific treatments provided by specialist practitioners such as surgery, acupuncture, spinal manipulation, injection therapy, massage, traction and electrical nerve stimulation will not be discussed as these are not readily available in the emergency setting.

A search of the following databases was conducted in March 2007 to find relevant systematic reviews and clinical trials: MEDLINE, CINAHL, Cochrane library, Clinical Evidence, Ovid EBMR along with an internet search to locate evidence based clinical practice guidelines. Key terms that were searched included 'back pain', 'sciatica', 'low back pain' and 'lumbar pain' in combination with key terms from each of the topics discussed below. Results were limited to English language and human studies.

\section{Results}

Following abstract screening to ensure the articles were relevant to the emergency setting and excluded chronic back pain and specialty settings 35 articles were included in the review. This comprised of 14 systematic reviews, 10 review articles, 6 international clinical guidelines, 4 randomised control trials and 1 cohort study.

\section{Discussion}

\section{Aetiology, prevalence and prognosis}

There are a number of causes of acute lower back pain including fractures, infection, tumours and conditions such as osteoarthritis, lumbar spondylitis, spinal canal stenosis and non specific causes. ${ }^{4}$ The precise aetiology may only be identified in $15 \%$ of cases; however this does not preclude commencement of effective treatment. ${ }^{4,5}$ Non specific back pain is the most common cause of low back pain comprising $95 \%$ of cases in primary care. ${ }^{4}$ The prevalence of fractures is estimated at $3-5 \%$ and these are usually associated with trauma, the elderly or corticosteroid users following minor trauma. ${ }^{4}$ Infection, tumours, Ankylosing Spondylitis and degenerative spinal conditions are all much more rare causes of lower back pain. ${ }^{4}$ Conditions such as osteoarthritis, lumbar spondylitis and spinal canal stenosis may be commonly found on imaging studies in asymptomatic patients and therefore may not be related to the cause of the patient's pain. ${ }^{6}$ Despite these conditions being more uncommon than non specific pain they present
Table 1 Red flags

- Features of Cauda Equina syndrome including urinary retention, faecal incontinence, widespread neurological symptoms and signs in the lower limb, including gait abnormality, saddle area numbness and a lax anal sphincter.

- Significant trauma

- Weight loss

- History of cancer

- Fever

- Intravenous drug use

- Steroid use

- Patient over 50 years

- Severe, unremitting night-time pain

- Pain that gets worse when lying down

Source: Ref. ${ }^{7}$.

serious pathology which must be excluded in all patients who present with lower back pain. A number of clinical guidelines have identified 'red flags' which should be identified in the initial patient assessment to exclude serious conditions that require further investigation or actions (see Table 1 ).

The prevalence of low back pain is difficult to determine as studies have utilised a variety of definitions of low back pain and the natural history of back pain fluctuates making classification difficult. ${ }^{8}$ Prevalence of back pain ranges from $15-45 \%$ with point prevalence of $30 \%$ reported by Andersson. ${ }^{9}$ Peak prevalence occurs between the ages of 35 and 55, with equal distribution between males and females. ${ }^{8,10}$ There is little data on the volume or impact of patients with back pain presenting to emergency departments, although given the high prevalence and incidence in the community back pain could be regarded as a relatively common presentation.

The natural course and prognosis of low back pain has been well documented. A systematic review conducted by Pengel et al. suggests pain and disability usually decrease within one month of the acute episode and continues to decrease more slowly over the following 12 months. ${ }^{11}$ People are also likely to have at least one recurrence in the twelve months following the acute episode. ${ }^{11} \mathrm{~A}$ small percentage of patients go on to develop chronic back pain following an acute episode.

Despite a large number of studies regarding prognosis in lower back pain there are difficulties in conducting systematic reviews due to the wide range of outcome measures and the lack of clear definitions in the field. For example acute back pain is variously defined in clinical practice guidelines as pain of less than six weeks or less than three months duration. ${ }^{7,8,10}$ Further confusing analysis are the wide range of outcome measures that have been investigated including pain, disability, prevention of recurrence and chronicity and impact on employment to name a few. ${ }^{4,8,10}$ Hayden et al. ${ }^{31}$ suggest minimum outcome measures of pain scores; functional status and general health should be included in all studies investigating lower back pain although this recommendation is not currently evident in the literature. To improve synthesis and application of the findings for clinician's clear definitions are required with an agreed set of relevant outcome measures. 


\section{Psychosocial and work factors}

Historically lower back pain has been considered a biomedical problem that can be cured through understanding of anatomic damage to the spine and management of pathological conditions. ${ }^{17,18}$ There has been discussion in the literature regarding a move away from the biomedical model to a biopsychosocial model where a more holistic approach to the patient is advocated and psychological, social and biological factors are considered in the treatment of back pain. ${ }^{17,19}$ Evidence is emerging of the importance of considering biopsychosocial factors in the initial assessment of patients with low back pain as predictors of risk for developing chronic back pain. ${ }^{18}$

Hoogendoorn et al. conducted a high quality systematic review evaluating the psychosocial factors of work and private life on the occurrence of back pain..$^{20}$ Low workplace support and low job satisfaction were both found to be associated with low back pain while the impact of other risk factors relating to work and private life are yet to be established. ${ }^{20}$ Pincus et al. found distress; depressive mood and somatizisation were all linked with a transition to chronic back pain in a systematic review looking specifically at psychological factors. ${ }^{21}$ Despite their inclusion in clinical practice guidelines as risk factors the review by Pincus et al. was not able to confirm other psychological factors such as fear avoidance and coping strategies were linked to development of chronic back pain and suggests further studies are required to classify the role of these factors in back pain. ${ }^{21}$ Since this review has been published a cohort study found fear avoidance and distress are associated with progression to chronic low back pain. ${ }^{22}$ Another systematic review found a number of work factors are important in progression to chronic low back pain and disability including job satisfaction, monotonous work, work relations, self rated work demands, self reported stress and perceived ability to work. ${ }^{23}$

The national guidelines reviewed provide a variety of approaches to assessing and evaluating psychosocial risk factors. The New Zealand Guideline Group and Prodigy advocate assessment for 'yellow flags' (see Table 2 ) as predictors of poor outcome and high risk for chronicity and disability with the New Zealand guidelines providing a structured questionnaire to evaluate patients risk level. ${ }^{7,8}$ While advocating the assessment and identification of 'yellow flags' the

Table 2 Yellow flags

Yellow flags indicate psychosocial barriers to recovery. They include:

- Belief that pain and activity are harmful

- 'Sickness behaviours' (like extended rest)

- Low or negative moods, social withdrawal

- Treatment that does not fit best practice

- Problems with claims and compensation

- History of back pain, time off, other claims

- Problems at work, poor job satisfaction

- Heavy work, unsociable hours

- Overprotective family or lack of support

Source: Ref. ${ }^{7}$.
Australian Acute Musculoskeletal Pain Guidelines Group suggests that while there is general agreement in the literature regarding their use further studies are required to determine the combination of psychosocial parameters that provide optimal predictive value. ${ }^{4}$ Clinicians should have an awareness of psychosocial factors from the first visit and consider undertaking a formal assessment of these parameters if pain is ongoing. ${ }^{10}$ Optimal management of patients with identified psychosocial concerns have not been established. ${ }^{24}$

\section{History and physical examination}

The aim of the history and examination is to exclude serious pathology (red flags) and define the clinical problem. A number of authors and national guidelines suggest back pain can be triaged into three groups; (1) serious spinal pathology, (2) nerve root pain/radicular pain and (3) Non-specific low back pain. ${ }^{10,12}$ An accurate history and physical examination is essential in performing this triage and determining the management strategy for all patients presenting with back pain to the emergency department.

van den Hoogen et al. conducted a systematic review of 36 studies to evaluate the accuracy of history taking in diagnosing lower back pain. The review found only moderate accuracy in history taking and physical examination in identifying red flag conditions that either required surgery or further investigation such as vertebral cancer and ankylosing spondylitis. ${ }^{13}$ There is generally a wide variation in the sensitivity and specificity reported for individual history items such as parasthesia and sciatica and examination tests such as the straight leg raise and testing of lower limb reflexes making it difficult to draw strong conclusions of the accuracy of any one test. ${ }^{12,13} \mathrm{~A}$ recently completed study investigating reliability of common clinical tests and questions for low back pain found only poor to fair reliability between clinicians (Physiotherapists in this study). ${ }^{14}$ No single test or component of the history is sensitive or specific enough to diagnose a specific disease although van den Hoogen et al. ${ }^{13}$ did find a combination of history and erythrocyte sedimentation rate is diagnostic of vertebral cancer. Other studies have also suggested combining tests improves the accuracy of the examination. ${ }^{12,15}$

An example of the difficulties associated with studies related to physical examination of back pain is highlighted by the findings of a systematic review of the passive straight leg raise. The passive straight leg raise is a common test used in clinical practice as an aid to diagnosing lower back pain. A positive straight leg raise test is thought to be associated with intervertebral disc herniation and nerve root problems amongst other theories of the pathophysiology and clinical significance of the test. ${ }^{15}$ The review found no consensus on how the test should be performed, the relevance of a positive finding or the implication of a positive or negative test on patient outcomes. ${ }^{15} \mathrm{~A}$ number of national guidelines however suggest a straight leg raise is the most important tool in identifying nerve root pain. ${ }^{8,10}$ This may be due to the high sensitivity (90\%) and low specificity (20\%) of the test; however this may lead to false positive findings and over investigation. ${ }^{8}$ The systematic review suggests the use of the passive straight leg raise test is limited while the guidelines contradict this finding directly. 
The findings of the studies and reviews reported above suggest a wide degree of variation in findings and importance of individual history and examination parameters. While each test or parameter alone is not indicative of a specific cause for back pain the clinician may investigate further through repeat examination, imaging, laboratory studies or referral to specialists if specific serious conditions cannot be excluded. ${ }^{13}$ Therefore a thorough history and examination is required to exclude serious conditions and raise suspicions of serious disease which require further investigation. Despite inconclusive evidence to support the validity and reliability of the history and physical examination in patients with lower back pain there is consensus among expert groups of the need for a focussed history and examination to occur in all patients presenting with back pain. ${ }^{4,8,10}$

The history should generally include a history of the site, distribution and quality of pain, along with the duration, frequency, intensity and onset of the pain; the presence of sciatica; any aggravating or relieving factors and the effect of activities of daily living. ${ }^{4}$ The physical examination should include inspection, palpation for spinal tenderness, range of motion, neurological signs such as ankle and knee reflexes, sensory exam, neuromotor deficits and specific tests such as the straight leg raise. ${ }^{4,10,13,16}$

\section{Imaging}

There is general consistency between the national guidelines and related literature that plain X-rays in non-specific lower back pain are not useful in diagnosis and should be restricted to patients with identified 'red flags'. ${ }^{10,12,25,26}$ No benefit has been found in patients who received early radiographs and a greater proportion of patients who received $X$-rays reported more pain at three months and had higher consultation rates than those who did not have X-rays. ${ }^{26}$ Patients who had Xrays were more satisfied with their care but no differences in outcomes were reported at nine months. ${ }^{26}$ The Kendrick et al. study was based in the primary care setting and included patients who had a minimum of six weeks of pain which is an area where clinicians may consider an X-ray appropriate given the duration of the pain. ${ }^{26}$ For patients over the age of fifty or with findings suggestive of systemic disease ('red flags') plain radiography and simple pathology tests can almost completely exclude serious conditions and therefore should be performed in this group of patients. ${ }^{10,12}$ The New Zealand guidelines ${ }^{7}$ suggest an appropriate approach may be to initiate plain X-rays and FBC and ESR if pain lasts longer than six weeks even in the absence of red flags.

The limitations of plain X-ray are the number of false positives identified. Conditions such as spondylitis, facet joint abnormalities and some congenital abnormalities are diagnosed on X-rays just as commonly in asymptomatic patients as those with back pain. ${ }^{12}$ Further confusing the use of radiographs is the reported false-negative results which for known vertebral cancer have been reported at $41 \% .{ }^{4}$ Patients are also exposed to the risks associated with radiation.

The use of Magnetic resonance imaging (MRI) and Computed Tomography (CT) in non-specific low back pain is negligible if red flags have not been identified. A systematic review by Jarvik and Deyo found MRI to have the greatest sensitivity and specificity for serious pathology despite sys- tematic biases in the studies included in the review. ${ }^{26} \mathrm{CT}$ and MRI were found to be similarly accurate in identifying herniated discs and spinal stenosis, while MRI more accurately diagnoses infections and malignancies. ${ }^{26}$ Jarvik and Deyo conclude that imaging before six weeks is unwarranted (unless systemic disease is suspected) as imaging is unlikely to identify the specific cause of the pain and specificity is limited by abnormal findings in normal adults. ${ }^{26}$ There is little benefit in substituting MRI for plain radiographs in the primary care setting due to the higher cost of the MRI and the low yield. ${ }^{4,27}$

\section{Therapies-medications}

Non-steroidal anti-inflammatory drugs (NSAIDs) are commonly used to treat acute episodes of lower back pain. There have been many clinical trials and at least one systematic review assessing the efficacy of NSAIDs on back pain. ${ }^{28}$ The most recent Cochrane systematic review involved 51 trials of which $31 \%$ were of high quality. ${ }^{28}$ Several weaknesses in methodology were found with the studies including inadequate randomisation procedures, unsatisfactory compliance and inadequate follow-up period. ${ }^{28}$ Despite this the review found strong evidence that NSAIDs compare favourably with placebo in relieving back pain at one week however the meta analysis has been criticised for involving RCT's with a mix of subjects with chronic and acute pain and patients with sciatica only. ${ }^{3,4,28}$ The Australian Acute Musculoskeletal Pain Guidelines Group concludes there is conflicting evidence of the effectiveness of NSAIDs versus placebo for treatment of acute low back pain. However Clinical Evidence, the New Zealand, United Kingdom and European guidelines all recommend the use of NSAIDs for acute lower back pain in combination with other medications. ${ }^{3,7,8,10}$

The Cochrane review found no difference in outcomes among individual NSAIDs although there have been no studies on the effectiveness of topical NSAIDs in the treatment of back pain. ${ }^{4,29}$ When compared with other drugs there was high level evidence that NSAIDs have a similar effect to opioid and paracetamol-opioid combination drugs. ${ }^{4}$ The risks associated with the use of NSAIDs include gastrointestinal bleeding and perforation. ${ }^{4}$

There are no randomised control trials assessing the effectiveness of paracetamol in acute low back pain. ${ }^{3,4}$ The European and UK guidelines suggest the use of paracetamol as a first line agent due to evidence of the effectiveness of paracetamol in the treatment of other musculoskeletal pain and the limited side effects. If paracetamol alone is not effective a combination medication containing a simple analgesia (Paracetamol) with a opioid (Codeine) may be added. ${ }^{10}$

The use of muscle relaxants (benzodiazepines) in the treatment of lower back pain is controversial despite evidence in three systematic reviews of the benefit of these agents in reducing pain. ${ }^{10}$ The New Zealand Guideline Group concluded that there is evidence of harm with the use of narcotics and diazepam while the Australian guideline on lower back pain suggests the evidence is conflicting and there are serious side effects associated with the use of muscle relaxants. ${ }^{4,7}$ The Cochrane review also suggests caution be used with benzodiazepines due to the significant 
increase in risk and adverse effects associated with muscle relaxants and also recommends further studies comparing these agents to NSAIDs and other analgesics. ${ }^{29}$ Due to these increased risks and uncertainty the UK and European guidelines recommend a short course of a muscle relaxant only if paracetamol and an NSAID combination fail to reduce pain. 8,10

There have been no randomised control trials evaluating the effectiveness of opioids compared with placebo in low back pain. ${ }^{4}$ There is however some evidence that opioids/combination opioids/paracetamol are comparable to NSAID's for the treatment of low back pain. ${ }^{4}$ The New Zealand guidelines recommend against using opioids due to the increased risk of adverse effects while other guidelines advocate the use of weak opioids when paracetamol and NSAID in combination have failed. ${ }^{8,16,30}$

\section{Therapies-exercise therapy and activity}

There are a number of exercise therapies and programs available with the aim of improving function and decreasing pain in patients with lower back pain. These programs include stretching and strengthening exercises, manual therapy and back schools. ${ }^{31}$ A systematic review of eleven randomised control trials conducted in 2005 found conflicting evidence of the benefits of exercise programs. ${ }^{32}$ Eight of the studies were of low quality and found no statistically significant differences between the control group (usual care or no treatment) and the exercise program, while one high quality study found usual care to be more effective than home-exercises. ${ }^{32}$ Hayden et al. state that the studies were generally of poor quality and in the majority of studies the only outcome measure was pain score. ${ }^{31}$ This systematic review has been criticised for 'lumping' all the various forms of exercise therapy into one review with a lack of description of the specific nature of the exercises studied. ${ }^{32}$ Not only does this limit the clinical relevance of attempting to recommend a specific exercise program to patients it also ensures a heterogeneous group of studies that should possibly not be compared in the first place. This suggests further studies are required with clearer outcome measures before recommending exercise programs to patients.

Bed rest has historically been used in the management of back pain although more recently there has been evidence suggesting this may not be beneficial. ${ }^{33}$ Results from at least six systematic reviews suggest bed rest may be worse than no treatment, advice to stay active and NSAID's. ${ }^{4}$ Hagen et al. is critical of the previous reviews as they evaluate bed rest in all types and causes of back pain questioning the validity of these results for clinicians. ${ }^{32}$ The latest Cochrane review concluded advice to stay active is more beneficial than bed rest while for patients with nerve root involvement (sciatica) there is no difference between bed rest and advice to stay active. ${ }^{33}$

One randomised control trial found patients having two days bed rest had significantly less sick leave compared with patients having seven days bed rest, although the study also found no difference in pain, functional level or recovery at three and twelve weeks. ${ }^{34}$ The Australian, UK and New Zealand guidelines recommend against bed rest for longer than two days while the European guidelines recommend against advising bed rest at all. ${ }^{4,7,8,30}$
Advice to stay active has also been recommended in a number of clinical guidelines for lower back pain. There is evidence staying active can reduce sick leave, pain levels and improve recovery and function compared to bed rest. ${ }^{24}$ Staying active and returning to work and normal activity is recommended in all the guidelines reviewed.

\section{Therapies-patient information}

Patient information may come in the form of verbal advice, written or electronic material. A Cochrane review in 2006 found much of the patient information regarding back pain is based on a biomedical model and include information that is out of date and not consistent with current back pain guidelines. ${ }^{35}$ The review concluded that there is high level evidence (based on five randomised control trials) that booklets increase knowledge compared with no intervention or usual care. ${ }^{35}$ Guidelines and the Cochrane review support printed information in conjunction with verbal advice and reinforcement from clinicians as an effective approach. 4,10,35 Van Tulder et al. suggest advice should include the following 'good prognosis, no need for X-rays, no underlying pathology, and stay active (10)'. The evidence supports a biopsychosocial approach in preference to biomedical content in delivering information. ${ }^{35}$ The limitations of the Cochrane systematic review included the wide range of information delivery methods and content included in the review, the small number of studies included and a heterogeneous population in terms of duration of lower back pain characteristics. Suggested content for advice for patients is included in Table 3 as a guide.

\section{Follow up}

No studies have specifically addressed an appropriate follow up period following an episode of non-specific low back pain however clinical guidelines suggest a range of one week to six weeks depending on improvement or ongoing

Table 3 Recommended patient information and advice

Specific content for advice includes

- Reinforcing the likely absence of serious disease when red flags are not present

- Hurt does not equal harm. Back pain is a mechanical problem not related to structural damage.

- Emphasise a good prognosis for low back pain. The majority of patients experience significant improvements in 2 to 4 weeks

- Bed rest is not recommended

- Light activity will not further injure the spine and helps speed recovery

- Progressive resumption of work and activity leads to better short term and long term outcomes

- Back pain can fluctuate in intensity and may recur.

- The person with back pain must accept shared responsibility for recovery with health professionals.

Source: Refs. ${ }^{8,16}$. 
symptoms. ${ }^{10}$ The United Kingdom clinical guideline suggests assessing the response to treatment at four weeks although it may be more appropriate for follow up to be flexible depending on the level of pain and associated symptoms. ${ }^{8,10}$ The New Zealand and United States guidelines recommend weekly reviews until symptoms are improving and the patient is managing the condition and has resumed normal activities. Follow up should include evaluation of psychosocial factors, nerve root pain, disability and risk factors for red flags. ${ }^{8}$

\section{Clinical implications and recommendations}

The evidence suggests back pain is a condition that can be safely managed in the emergency setting without the need for expensive imaging studies or other investigations beyond a thorough medical history and examination. The objective of the assessment is to exclude serious pathology and determine the nature of the pain. Psychosocial factors should be considered as predictors of progression to chronic back pain. Treatment is aimed at minimising pain and disability and returning patients to normal activity. Simple analgesia such as paracetamol and NSAID's are recommended in combination with advice to remain active and avoid bed rest. Adequate follow up should be arranged preferably with the patient's general practitioner. For emergency departments the guidelines suggest appropriately identified patients may not need to be delayed in the department waiting for imaging studies and other tests which may increase their length of stay.

The body of evidence in regards to the assessment and treatment of non specific lower back pain is growing with many high quality systematic reviews, prospective cohort studies and randomised control trials published. The quality of the literature has been criticised in some of these systematic reviews with regard to inconsistencies in definitions, heterogeneous samples including "chronic" and "acute" subjects and methodological biases. ${ }^{36}$ Despite these criticisms there is general agreement internationally in the findings of the literature expressed through clinical guidelines that advocate a biopsychosocial and active approach to the management of acute low back pain. Further work is required to ensure the evidence is introduced into practice and ensure consistency between practitioners in the approach to management of this group of patients.

Limitations of this review are that only studies in the English language were included. The use of clinical guidelines may also be criticised as a 'lesser' level of evidence due to the greater emphasis on consensus expert opinion. The guidelines cited in this review are all nationally recognised guidelines based on extensive reviews of the literature and therefore are considered valid, although clinicians must be aware international guidelines may not always be adaptable to the Australian context.

\section{Conclusion}

This review provides a summary of an approach to management of patients presenting to the emergency department with lower back pain based on the available evidence. While there are limitations with the literature and this review the evidence is clear regarding many aspects of management. Further research is required to determine the clinical validity and reliability of a number of history items and physical examination tests including the straight leg raise. The importance of psychosocial factors in the clinical setting requires further research particularly the management of patients who have identified risk factors for progression to chronicity. It is hoped this review will improve clinical practice and aid in dissemination of research findings. This review can also be used as the basis for local clinical guideline development and associated standards and quality indicator development with the aim of improving practice through the use of an evidence based approach.

\section{Competing Interests}

None declared.

\section{Funding}

None declared.

\section{References}

1. Koes BW, van Tulder MW. Low back pain (acute). Available at: http://gateway.ut.ovid.com/gw1/ovidweb.cgi. Accessed March 17, 2007.

2. D'Arcy Y. Treatment strategies for low back pain relief. Nurse Pract 2006;31(4):16-25.

3. Koes BW, van Tulder MW. Low back pain (acute). Available at: http://gateway.ut.ovid.com/gw1/ovidweb.cgi. Accessed March 31, 2007.

4. Australian Acute Musculoskeletal Pain Guidelines Group. Evidence-based management of acute musculoskeletal pain. Bowen Hills: Australian Academic Press Pty. Ltd.; 2003.

5. Lehrich J, Katz J, Sheon R. Approach to the diagnosis and evaluation of low back pain in adults. Available at: http://www. utdol.com/utd/content/topic.do?topickey=spinaldi/2946\& view=print. Accessed March 17, 2007.

6. van Tulder MW, Assendelft WJJ, Koes BW, Bouter LM. Spinal radiographic findings and non specific low back pain: a systematic review of observational studies. Spine 1997;22:427-34.

7. Accident Compensation Corporation, New Zealand Guidelines Group. New Zealand Acute Low Back Pain Guide. Available at: http://www.nzgg.org.nz/guidelines/dsp_guideline_popup. cfm?\&guidelinelD=72. Accessed March 22, 2007.

8. Prodigy Guidance. Back Pain - lower. Available at: http:// www.prodigy.nhs.uk/prodigyknowledge/Assets/CommonUICControls/Print.aspx. Accessed March 17, 2007.

9. Andersson GBJ. Epidemiological features of chronic low back pain. Lancet 1999;354(9178):581-5.

10. Van Tulder MW, Becker A, Bekkering T, Breen AC, Gil del Real MT, Hutchinson A, Koes B, Laerum E and Mealmivaara A. European guidelines for the management of acute non specific low back pain in primary care. Available at: http:// www. backpaineurope.org/web/html/wg1_results.html. Accessed March 22, 2007.

11. Pengel HM, Herbert RD, Maher CG, Refshauge KM. Acute low back pain: systematic review of its prognosis. BMJ 2003;327:323-8.

12. Jarvik JG, Deyo RA. Diagnostic evaluation of low back pain with emphasis on imaging. Ann Int Med 2002;137(7):586-97.

13. van den Hoogen HM, Koes BW, van Eijk JT, Bouter LM. On the accuracy of history, physical examination, and erythro- 
cyte sedimentation rate in diagnosing low back pain in general practice. A criteria-based review of the literature. Spine 1995;20(3):318-27.

14. McCarthy CJ, Gittins M, Roberts C, Oldham JA. The reliability of the clinical tests and questions recommended in international guidelines for low back pain. Spine 2007;32(8):921-6.

15. Rebain R, Baxter GD, McDonough S. The passive straight leg raising test in the diagnosis and treatment of lumbar disc herniation: a survey of United Kingdom osteopathic opinion and clinical practice. Spine 2003;28(15):1717-24.

16. Institute for Clinical Systems Improvement. Adult low back pain. Available at: www.icsi.org. Accessed March 30, 2007.

17. Waddell G. A new clinical model for the treatment of low-back pain. Spine 1987;12(7):632-44.

18. Borkan J, Van Tulder MW, Reis S, Schoene ML, Croft P, Hermoni D. Advances in the field of low back pain in primary care. A report from the fourth international forum. Spine 2002;27(5):E128-32.

19. Borge JA, Leboeuf-Yde C, Lothe J. Prognostic values of physical examination findings in patients with chronic low back pain treated conservatively: a systematic literature review. J Manip Physiol Ther 2001;24(4):292-5.

20. Hoogendoorn WE, van Poppel MN, Bongers PM, Koes BW, Bouter LM. Systematic review of psychosocial factors at work and private life as risk factors for back pain. Spine 2000;25(16):2114-25.

21. Pincus T, Burton K, Vogel S, Field AP. A systematic review of psychological factors predictors of chronicity/disability in prospective cohorts of low back pain. Spine 2002;27(5):E109-20.

22. Grotle M, Brox JI, Veierod MB, Glomsrod B, Lonn JH, Vollestad NK. Clinical course and prognostic factors in acute low back pain. Spine 2005;30(8):976-82.

23. Linton SJ. A review of psychosocial risk factors in back and neck pain. Spine 2000;25:1148-56.

24. Koes B, Van Tulder MW, Thomas S. Diagnosis and treatment of low back pain. BMJ 2006;332:1430-4.

25. Kendrick D, Fielding K, Bentley E, Kerslake R, Miller P, Pringle $M$. Radiography of the lumbar spine in primary care patients with low back pain: randomised controlled trial. BMJ 2001;322(7283):400-5.
26. Kendrick D, Fielding K, Bentley E, Miller P, Kerslake R, Pringle $M$. The role of radiography in primary care patients with low back pain of at least 6 weeks duration: a randomised (unblinded) controlled trial. Health Technol Assess 2001;5(30):169.

27. Gilbert FJ, Grant AM, Gillan MG, Vale L, Scott NW, Campbell ML, Wardlaw D, Knight D, Mclntosh E, Porter RW. Does early imaging influence management and improve outcome in patients with low back pain? A pragmatic randomised controlled trial. Health Technol Assess 2004;8(17):1131.

28. van Tulder MW, Scholten RJ, Koes BW, Deyo RA. Nonsteroidal anti-inflammatory drugs for low back pain: a systematic review within the framework of the Cochrane Collaboration Back Review Group. Spine. 2000; 25(19):2501-13.

29. van Tulder MW, Touray T, Furlan AD, Solway S, Bouter LM. Cochrane Back Review, G. Muscle relaxants for nonspecific low back pain: a systematic review within the framework of the Cochrane collaboration. Spine 2003;28(17):197892.

30. van Tulder MW, Malmivaara A, Esmail R, Koes B. Exercise therapy for low back pain. A systematic review within the framework of the Cochrane Collaboration Back Review Group. Spine 2000;25(21):2784-96.

31. Hayden JA, van Tulder MW, Malmivaara A, Koes BW. Exercise therapy for treatment of non-specific low back pain. Cochrane Database Syst Rev 2005:3.

32. Hagen KB, Jamtvedt G, Hilde G, Winnem MF. The updated cochrane review of bed rest for low back pain and sciatica. Spine 2005;30(5):542-6.

33. Deyo RA, Diehl AK, Rosenthal M. How many days of bed rest for acute low back pain? N Eng J Med 1986;315:1064-70.

34. Henrotin YE, Cedraschi C, Duplan B, Bazin T, Duquesnoy B. Information and low back pain management: a systematic review. Spine 2006;31(11):15.

35. Cherkin DC. Primary care research on low back pain: the state of the science. Spine 1998;23(18):1997-2002.

36. Gray JAM. Evidence based healthcare. In: How to make health policy and management decisions. 2nd ed. Loanhead: Churchill Livingstone; 2001. 